\title{
Lagrangian Finite Element Method with Nodal Integration for Fluid-Solid Interaction
}

\author{
Alessandro Franci
}

Received: date / Accepted: date

\begin{abstract}
This work presents a fully Lagrangian Finite Element Method (FEM) with nodal integration for the simulation of Fluid-Structure Interaction (FSI) problems. The Particle Finite Element Method (PFEM) is used to solve the incompressible fluids and to track their evolving free surface, while the solid bodies are modeled with the standard FEM. The coupled problem is solved through a monolithic approach to ensure a strong FSI coupling. Accuracy and convergence of the proposed nodal integration method are proved against several benchmark tests, involving complex interactions between unsteady free-surface fluids and solids undergoing large displacements. A very good agreement with the numerical and experimental results of the literature is obtained. The numerical results of the nodal integration algorithm are also compared to those given by a standard Gaussian method and their upperbound convergent behavior is also discussed.
\end{abstract}

Keywords Nodal Integration · PFEM · FSI • Free-Surface

International Center for Numerical Methods in Engineering (CIMNE), Universitat Politecnica de Catalunya

Carrer Gran Capitan, UPC Campus Nord, Barcelona

E-mail: falessandro@cimne.upc.edu

\section{Introduction}

This paper presents a Lagrangian Finite Element Method (FEM) with nodal integration for the simulation of Fluid-Structure Interaction (FSI) problems, also in the presence of free-surface fluid flows. The large motion and deformation of the fluid, as well as the detection of the fluid-solid interface, are handled with the Particle Finite Element Method (PFEM) $[16,28,17]$, a well-assessed numerical technique that combines the Lagrangian FEM solution of the governing equations with an efficient remeshing strategy. On the contrary, the solid parts of the computational domain are not involved in the remeshing operations and they are solved with a standard FEM. The strongly coupled solution of the FSI problem is obtained with the monolithic strategy called Unified formulation $[17,13]$. This PFEM-FEM model yields to a conforming-mesh FSI algorithm, i.e. the contact elements (those elements connecting solid and fluid domains) have overlapped fluid and solid nodes at the interface. The resulting mesh is used to discretize the governing equations as in a standard elemental FEM, but their integration is performed over the nodal patches and not over the elements, and the stresses/strains are defined at the nodes and not at the Gauss points. The integration pro- 
cedure used in this work is based on the nodal integration scheme for FEM presented in [7].

There are several points of interest in the application of a coupled PFEM-FEM strategy with nodal integration to FSI problems.

In the recent work [10], it has been shown that PFEM with nodal integration (in the following, nodal PFEM) presents some important skills in the framework of free-surface fluid analysis. In particular, if compared to the classic PFEM with elemental integration (in the following, elemental PFEM), the nodal PFEM showed to suffer less from mesh distortion, to give a more accurate stress field, and to need fewer iterations to converge. However, for the same mesh, it was shown that the elemental PFEM is more accurate in the solution of the unknown variables (nodal velocities and pressures) and has a reduced computational cost to build and solve the linear system.

In non-linear solid mechanics, nodal PFEM has an important advantage versus elemental PFEM. In fact, in a nodal integration method, remapping operations of historical variables during the remeshing step are avoided because all variables, including stresses and strains, are stored at the nodes. This is crucial for the conservation and accuracy of the historical variables because interpolation errors are completely avoided. However, it is important to remark that this does not mean that a nodal integration method is completely immune from the re-generation of the mesh. Indeed, as for the standard elemental PFEM [9], also a nodal approach may suffer from the perturbation generated by the changes of elemental connectivity and topology (e.g. the creation or elimination of new boundary elements). The response of a solid nodal PFEM to these remeshing situations is an issue that deserves a deep specific study and it is considered out of the scope of the present work.

Another well-known property of FEM formulations with nodal integration (in the following, nodal FEM) is that they are expected to give an upper bound solution to the elastic problem [21, 20,8]. In [10], an analogous behav- ior was also found for the nodal PFEM in the context of fluid dynamics problems. In other words, for a given mesh, a nodal FEM gives a softer (in fluid dynamics, less viscous) solution than the expected one, contrary to a FEM with elemental integration (in the following, elemental FEM), which converges to the solution from below. In theory, this dual property enables to get a range of possible solutions by combining the results of an elemental FEM (lower-bound solution) and those of a homologous nodal FEM (upper-bound solution). This way, one could obtain an indicative prediction of the expected solution also using relatively coarse meshes. This feature could be very helpful for some demanding fluid, solid, or FSI problems, where fine meshes cannot be employed due to computational cost reasons.

The definition of stresses and strains at the nodes gives another important advantage to nodal FEM models. In fact, in a nodal method, the one-field approach (e.g. velocity formulation) is expected to give the same solution as a mixed method using pressure as the additional nodal unknown (e.g. velocity-pressure formulation), at least if the problem is not fully-incompressible and does not require stabilization. This feature is particularly useful in the framework of FSI analysis, where mixed conforming-mesh methods with Gaussian integration, such as the elemental PFEM formulations $[17,13]$, require the duplication of the pressure degrees of freedom at the interface nodes. This complication is avoided in a nodal integration FEM framework because the nodal pressures are used only for the solution of the incompressible fluids and not to solve the compressible or quasi-incompressible solids.

All the described properties of the nodal integration algorithm explain the growing interest in applying this technique to a PFEM framework. The first contribution in this field was given by $[35,34]$ with their so-called Smoothed Particle Finite Element Method (SPFEM). The authors took inspiration from the Smoothed Finite Element Method (SFEM) [19,26,38, 18], and applied successfully their PFEM with nodal 
integration to geomechanics applications. More recently, [10] explored the nodal PFEM in the context of unsteady free-surface fluid dynamics. The present work wants to follow this investigation line and, for the first time in the literature, it analyzes the application of a nodal PFEM to FSI problems. In fact, despite the large number of published PFEM formulations for FSI analysis (e.g. [17,24,4,23]), still, there does not exist a similar approach in the literature. Note that, also in the family of SFEM methods, there are only a few examples of formulations for FSI (e.g. [39,38,37]), especially in presence of free-surface fluids.

From a broader perspective, this work should be considered as a further step towards a unified nodal PFEM formulation for non-linear fluid and solid mechanics.

The paper is structured as follows. In Section 2, first, the governing equations of the problem are presented, and then, the fully discretized form of the problem is derived in a nodal integration framework. In Section 3, the fundamentals of PFEM are recalled and the algorithm to detect the fluid-solid interface is explained. Section 4 presents the linearized form of the problem and it describes the FSI solution algorithm. The validation examples are presented in Section 5. Finally, Section 6 gives the concluding remarks and presents the future research lines associated with this work.

\section{Governing equations}

In the spirit of the Unified method for FSI [17, 13], solid and fluid formulations are here presented together. The governing equations of the problem are the momentum balance and the mass conservation and they are formulated in an Updated Lagrangian framework, as in the standard PFEM [16].

Calling $\Omega$ the updated/deformed domain and being $(0, T)$ the time interval, the momentum balance equations read

$\rho \dot{\boldsymbol{v}}-\nabla \cdot \boldsymbol{\sigma}-\boldsymbol{b}=\mathbf{0} \quad$ in $\Omega \times(0, T)$ where $\rho$ is the density of the material, $\dot{v}$ is the time derivative of the velocity vector, $\sigma$ is the Cauchy stress tensor and $\boldsymbol{b}$ are the body forces per unit of volume.

The mass conservation, or continuity, equation is written as $[29,12]$

$\frac{1}{\kappa} \dot{p}-d_{v}(\boldsymbol{v})=\mathbf{0} \quad$ in $\Omega \times(0, T)$

where $\kappa$ is the bulk modulus of the material and the volumetric deformation rate $d_{v}$ is defined as

$d_{v}(\boldsymbol{v})=\nabla \cdot \boldsymbol{v}=\operatorname{trace}(\boldsymbol{d})$

where the deformation rate $\boldsymbol{d}$ is computed from the velocity as

$\boldsymbol{d}=\frac{1}{2}\left(\nabla \boldsymbol{v}+[\nabla \boldsymbol{v}]^{T}\right)$

The system of Eqs. $(1$ - 2) must be completed with appropriate initial and boundary conditions.

On the Dirichlet $\left(\Gamma_{v}\right)$ and Neumann $\left(\Gamma_{t}\right)$ boundaries, the following conditions are imposed

$$
\begin{array}{lr}
\boldsymbol{v}-\hat{\boldsymbol{v}}=0 & \text { on } \Gamma_{v} \\
\boldsymbol{\sigma} \cdot \boldsymbol{n}-\hat{\boldsymbol{t}}=0 & \text { on } \Gamma_{t}
\end{array}
$$

being $\hat{\boldsymbol{v}}$ and $\hat{\boldsymbol{t}}$ the prescribed velocities and tractions, respectively, and $\boldsymbol{n}$ the outgoing normal vector to the domain boundaries.

\subsection{Galerkin approximation}

Following a standard Galerkin approach, Eqs.(12) are integrated over the computational domain and each term is multiplied by test functions.

After applying the divergence theorem and using the vector test functions $\boldsymbol{w}$, the weak form of momentum equations Eq.(1) reads

$$
\begin{aligned}
& \int_{\Omega} \rho \boldsymbol{w} \cdot \dot{\boldsymbol{v}} d \Omega+\int_{\Omega} \boldsymbol{d}(\boldsymbol{w}): \boldsymbol{\sigma} d \Omega \\
& -\int_{\Omega} \boldsymbol{w} \cdot \boldsymbol{b} d \Omega-\int_{\Gamma_{t}} \boldsymbol{w} \cdot \hat{\boldsymbol{t}} d \Gamma=\mathbf{0}
\end{aligned}
$$


Analogously, using the scalar test function $q$, the following weak form of the continuity equation (Eq.(2)) is obtained

$\int_{\Omega} \frac{1}{\kappa} q \dot{p} d \Omega-\int_{\Omega} q d_{v} d \Omega=0$

As in the standard FEM, the computational domain $\Omega$ is discretized over a finite elements mesh composed by triangles (tetrahedra in threedimensions). Linear shape functions $\boldsymbol{N}$ are used to interpolate the velocity and pressure fields, as well as the test functions, as follows

$$
\begin{array}{ll}
\boldsymbol{v}(x, t)=\boldsymbol{N}(x) \overline{\boldsymbol{v}}(t) & \boldsymbol{w}(x, t)=\boldsymbol{N}(x) \overline{\boldsymbol{w}}(t) \\
p(x, t)=\boldsymbol{N}(x) \overline{\boldsymbol{p}}(t) & q(x, t)=\boldsymbol{N}(x) \overline{\boldsymbol{q}}(t)
\end{array}
$$

Note that the upper symbol $(\bar{\cdot})$ that appears in Eq.(9) denotes a nodal variable. This convention will be also used for the rest of the derivation.

Combining Eqs.(9) with Eqs.(7-8), the weak forms of the linear momentum and continuity equations transform into

$$
\begin{gathered}
\int_{\Omega} \rho \boldsymbol{N}^{T} \boldsymbol{N} d \Omega \dot{\overline{\boldsymbol{v}}}+\int_{\Omega} \boldsymbol{B}^{T} \boldsymbol{\sigma} d \Omega \\
-\int_{\Omega} \boldsymbol{N}^{T} \boldsymbol{b} d \Omega-\int_{\Gamma_{t}} \boldsymbol{N}^{T} \hat{\boldsymbol{t}} d \Gamma=\mathbf{0} \\
\int_{\Omega} \frac{1}{\kappa} \boldsymbol{N}^{T} \boldsymbol{N} d \Omega \dot{\overline{\boldsymbol{p}}}-\int_{\Omega} \boldsymbol{N}^{T} d v d \Omega=\mathbf{0}
\end{gathered}
$$

where the operator $\boldsymbol{B}$ used in Eq.(10) is defined such that [2]

$$
\begin{aligned}
\boldsymbol{d}(\boldsymbol{x}, t) & =\boldsymbol{B}(\boldsymbol{x}) \overline{\boldsymbol{v}}(t) \\
& =\frac{1}{2}\left(\nabla \boldsymbol{v}(\boldsymbol{x}, t)+[\nabla \boldsymbol{v}(\boldsymbol{x}, t)]^{T}\right)
\end{aligned}
$$

\subsection{Spatial integration}

In this approach, the weak form (Eqs.(10-11)) is integrated numerically over the nodal patches of the triangular mesh. In the bulk, the area of the nodal patch is the overall area of all neighboring elements, see the graphical representation in Figure 1a. Instead, for a node $I$ belonging to the fluid-solid interface Figure 1b, two different nodal areas must be defined, one for the fluid part $\left(\Omega_{F}^{I}\right)$ and one for the solid one $\left(\Omega_{S}^{I}\right)$. In both cases, the nodal patch area $\Omega^{I}$ of node I representing the material $m$ is defined as

$\Omega_{m}^{I}=\sum_{\alpha=1}^{n e_{m}^{I}} \frac{\Omega_{m}^{\alpha}}{3}$

where $\Omega_{m}^{\alpha}$ is the area of the neighbor element $\alpha$ of material $m$ and $n e_{m}^{I}$ is the number of elements of the same material sharing node $I$. The material of the elements is given by the material of the shared nodes (interface nodes have both materials definitions).

(a) Internal node $I$

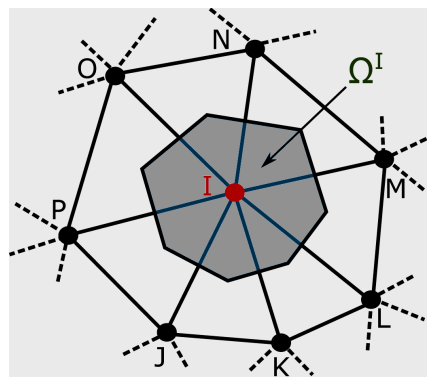

(b) Node $I$ at the inteface

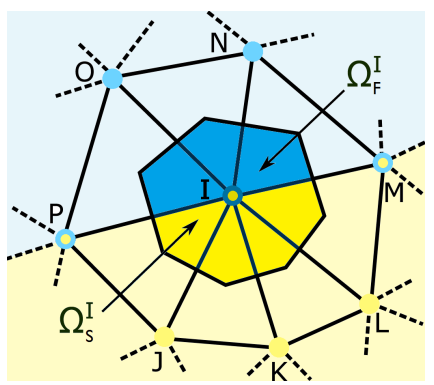

Fig. 1: Graphical representation of nodal patch. (a) patch area $\Omega^{I}$ of an internal node I. (b) Fluid and solid patch areas $\left(\Omega_{F}^{I}\right.$ and $\Omega_{S}^{I}$, respectively) of a node $I$ located at the fluid-solid interface.

Considering non-overlapping domains, nodal and elemental areas are defined such that

$\int_{\Omega_{m}} d \Omega=\sum_{I=1}^{N N_{m}} \Omega^{I_{m}}=\sum_{\alpha=1}^{N E_{m}} \Omega^{\alpha_{m}}$ 
where $N N_{m}$ and $N E_{m}$ are the total number of nodes and elements of the mesh of material $m$.

For the sake of simplicity and to lighten the notation, in the following, the sub-index specifying the node material is omitted and the derivation of the proposed nodal formulation will be carried on for a general material. Note that this is in line with the spirit of the Unified formulation for fluid and solid mechanics $[15,13]$. The sub-indices $f$ and $s$ specifying the fluid and solid parts, respectively, will be introduced into the formulation only when strictly necessary.

Using the nodal partition of the computational domain, the linear momentum equations (Eq.(10)) are rewritten as

$$
\begin{aligned}
& \sum_{I=1}^{N N} \int_{\Omega^{I}} \rho N^{I} N^{I} d \Omega \dot{\overline{\boldsymbol{v}}}^{I}+\sum_{I=1}^{N N} \int_{\Omega^{I}}\left[\overline{\boldsymbol{B}}^{I}\right]^{T} \boldsymbol{\sigma} d \Omega \\
& -\sum_{I=1}^{N N} \int_{\Omega^{I}} N^{I} \boldsymbol{b} d \Omega-\sum_{I=1}^{N N} \int_{\Gamma_{t}^{I}} N^{I} \hat{\boldsymbol{t}} d \Gamma=\mathbf{0}
\end{aligned}
$$

where symbol $\sum$ should be interpreted as the standard FEM assembly operator, and the nodal $\overline{\boldsymbol{B}}$ is computed for a generic node $I$ as

$$
\begin{aligned}
& \overline{\boldsymbol{B}}^{I}= \\
& {\left[\begin{array}{cccccccc}
N_{1, x}^{I} & 0 & \ldots & N_{I, x}^{I} & 0 & \ldots & N_{n n^{I}, x}^{I} & 0 \\
0 & N_{1, y}^{I} & \ldots & 0 & N_{I, y}^{I} & \ldots & 0 & N_{n n^{I}, y}^{I} \\
N_{1, y}^{I} & N_{1, x}^{I} & \ldots & N_{I, y}^{I} & N_{I, x}^{I} & \ldots & N_{n n^{I}, y}^{I} & N_{n n^{I}, x}^{I}
\end{array}\right]}
\end{aligned}
$$

Following [7], the derivatives of the shape functions in matrix $\bar{B}$ are computed from the elemental derivative of the shape functions as

$N_{J, j}^{I}=\frac{1}{A^{I}} \sum_{\alpha=1}^{n e^{I}} N_{J, j}^{\alpha} \frac{\Omega^{\alpha}}{3}$

Exploiting the linearity of the shape functions and being them equal to one at the nodes, the following nodal integrated form of the linear momentum is obtained

$$
\begin{aligned}
& \sum_{I=1}^{N N} \rho \Omega^{I} \dot{\overline{\boldsymbol{v}}}^{I}+\sum_{I=1}^{N N}\left[\overline{\boldsymbol{B}}^{I}\right]^{T} \overline{\boldsymbol{\sigma}}^{I} \Omega^{I} \\
& -\sum_{I=1}^{N N} \boldsymbol{b}^{I} \Omega^{I}-\sum_{I=1}^{N N} \alpha_{\Gamma}^{I} \hat{\boldsymbol{t}}^{I} \Gamma^{I}=\mathbf{0}
\end{aligned}
$$

where $\Gamma^{I}$ is the nodal contour length, $\alpha_{\Gamma}^{I}$ is equal to one if the node $I$ belongs to a Neumann boundary and equal to zero otherwise.

After analogous operations, the semi-discretized form of the continuity equation (Eq.(11)) is computed as

$\sum_{I=1}^{N N} \frac{1}{\kappa} \Omega^{I} \dot{\bar{p}}^{I}-\sum_{I=1}^{N N} \bar{d}_{v}^{I} \Omega^{I}=0$

where the nodal volumetric deformation rate $\bar{d}_{v}^{I}$ is computed from the nodal deformation rate tensor $\overline{\boldsymbol{d}}$ as

$\bar{d}_{v}^{I}=\operatorname{trace}\left(\overline{\boldsymbol{d}}^{I}\right)$

with

$\overline{\boldsymbol{d}}^{I}=\overline{\boldsymbol{B}}^{I} \overline{\boldsymbol{v}}^{n n^{I}}$

where vector $\overline{\boldsymbol{v}}^{\boldsymbol{n} \boldsymbol{n}^{\boldsymbol{I}}}$ contains the velocity components of node $I$ and those of its neighbor nodes. In $2 \mathrm{D}$, its size is $2 n n^{I}$, where $n n^{I}$ is the number of neighbors of node $I$ plus one.

\subsection{Time integration}

In order to obtain the fully discretized form of problem Eq.(18-19), the time derivative of nodal velocities and pressure must be defined.

For a generic time interval $\left[{ }^{n} t ;{ }^{n+1} t\right]$ of $\mathrm{du}-$ ration $\Delta t$, the nodal accelerations $\dot{\overline{\boldsymbol{v}}}$ are obtained using a second order Newmark scheme as [27]

$$
{ }^{n+1} \dot{\overline{\boldsymbol{v}}}=\frac{2}{\Delta t}\left({ }^{n+1} \overline{\boldsymbol{v}}-{ }^{n} \overline{\boldsymbol{v}}\right)-{ }^{n} \dot{\overline{\boldsymbol{v}}}
$$

Combining Eq.(22) and Eq.(18), the fully discretized form of the linear momentum equations at time ${ }^{n+1} t$ can be written as

$$
{ }^{n+1} \boldsymbol{R}_{\boldsymbol{m}}:={ }^{n+1} \boldsymbol{F}^{\boldsymbol{d}}+{ }^{n+1} \boldsymbol{F}^{\boldsymbol{\sigma}}-{ }^{n+1} \boldsymbol{F}^{\boldsymbol{e}}=\mathbf{0}
$$


where $\boldsymbol{F}^{\boldsymbol{d}}, \boldsymbol{F}^{\boldsymbol{\sigma}}$, and $\boldsymbol{F}^{\boldsymbol{e}}$ are the dynamic, internal and external equivalent nodal forces vector, respectively, and they are computed as follows

$$
\begin{aligned}
{ }^{n+1} \boldsymbol{F}^{\boldsymbol{d}}= & \sum_{I=1}^{N N} \rho\left[\frac{2}{\Delta t}\left({ }^{n+1} \overline{\boldsymbol{v}}-{ }^{n} \overline{\boldsymbol{v}}\right)-{ }^{n} \dot{\overline{\boldsymbol{v}}}\right]{ }^{n+1} \Omega^{I} \\
{ }^{n+1} \boldsymbol{F}^{\boldsymbol{\sigma}}= & \sum_{I=1}^{N N}\left[\overline{\boldsymbol{B}}^{I}\right]^{T}{ }^{n+1} \overline{\boldsymbol{\sigma}}^{I}{ }^{n+1} \Omega^{I} \\
{ }^{n+1} \boldsymbol{F}^{\boldsymbol{e}=} & \sum_{I=1}^{N N}{ }^{n+1} \boldsymbol{b}^{I}{ }^{n+1} \Omega^{I} \\
& -\sum_{I=1}^{N N} \alpha_{\Gamma}^{I n+1} \hat{\boldsymbol{t}}^{I}{ }^{n+1} \Gamma^{I}
\end{aligned}
$$

On the other hand, the time derivative of the pressure is computed as

${ }^{n+1} \dot{\bar{p}}=\frac{1}{\Delta t}\left({ }^{n+1} \bar{p}-{ }^{n} \bar{p}\right)$

Analogously to what has been done for the linear momentum equations, the fully discretized form of the continuity equation at time ${ }^{n+1} t$ is written in the following form

${ }^{n+1} \boldsymbol{R}_{\boldsymbol{c}}:={ }^{n+1} \boldsymbol{F}^{\boldsymbol{p}}-{ }^{n+1} \boldsymbol{F}^{\boldsymbol{v}}=\mathbf{0}$

being

$$
\begin{aligned}
{ }^{n+1} \boldsymbol{F}^{\boldsymbol{p}} & =\sum_{I=1}^{N N} \frac{1}{\kappa \Delta t}\left({ }^{n+1} \bar{p}-{ }^{n} \bar{p}\right){ }^{n+1} \Omega^{I} \\
{ }^{n+1} \boldsymbol{F}^{\boldsymbol{v}} & =\sum_{I=1}^{N N}{ }^{n+1} \bar{d}_{v}^{I}{ }^{n+1} \Omega^{I}
\end{aligned}
$$

\subsection{Stabilization}

For incompressible materials, the formulation is stabilized in order to circumvent the unfulfillment of the inf - sup condition [3]. In this work, the same stabilization procedure presented in [10] and based on the Finite Increment Calculus (FIC) stabilization technique [27] is used.
The FIC stabilization terms affect only the continuity equation (Eq.(26)), while the momentum equation (Eq.(23)) is left unchanged.

For the incompressible parts of the domain (in this work, the fluid parts only), Eq.(26) is modified by adding the stabilization term $\boldsymbol{F}^{\boldsymbol{\tau}}$ as follows

${ }^{n+1} \boldsymbol{R}_{\boldsymbol{c}}:={ }^{n+1} \boldsymbol{F}^{\boldsymbol{p}}-{ }^{n+1} \boldsymbol{F}^{\boldsymbol{v}}+{ }^{n+1} \boldsymbol{F}^{\boldsymbol{\tau}}=\mathbf{0}$

with

${ }^{n+1} \boldsymbol{F}^{\boldsymbol{\tau}}=\tau\left(\boldsymbol{L}^{n+1} \overline{\boldsymbol{p}}+\boldsymbol{M}_{\Gamma}{ }^{n+1} \overline{\boldsymbol{p}}+{ }^{n+1} \boldsymbol{f}_{p}\right)$

where the stabilization parameter $\tau$ is computed as [27]

$\tau=\left(\frac{8 \mu_{f}}{h^{2}}+\frac{2 \rho}{\delta}\right)^{-1}$

being $h$ and $\delta$ are characteristic distances in space and time [27].

Matrices $\boldsymbol{L}, \boldsymbol{M}_{\Gamma}$ and $\boldsymbol{f}_{p}$ of Eq.(29) are defined elementally and integrated for each fluid element $e$ as

$\boldsymbol{L}^{e}=\int_{\Omega^{e}}\left[\boldsymbol{\nabla} \mathbf{N}^{e}\right]^{T} \nabla \mathbf{N}^{e} d \Omega$,

$\left[M_{\Gamma}^{I J}\right]^{e}=\int_{\Gamma_{t}^{e}} \frac{2}{h_{n}} N^{I} N^{J} d \Gamma$

and

$$
\begin{aligned}
{\left[f_{p}^{I}\right]^{e}=} & \int_{\Gamma_{t}^{e}} N^{I}\left[\rho \dot{v}_{n}-\frac{2}{h_{n}}\left(2 \mu_{f} d_{n}-\hat{t}_{n}\right)\right] d \Gamma \\
& -\int_{\Omega^{e}}\left[\nabla \mathbf{N}^{e}\right]^{T} \boldsymbol{b} d \Omega
\end{aligned}
$$

where

$\boldsymbol{\nabla} \mathbf{N}^{e}=\left[\begin{array}{ccc}N_{1, x}^{e} & N_{2, x}^{e} & N_{3, x}^{e} \\ N_{1, y}^{e} & N_{2, y}^{e} & N_{3, y}^{e}\end{array}\right]$

and $h_{n}$ is a characteristic lenght of the element, $\dot{v}_{n}$ and $d_{n}$ and $\hat{t}_{n}$ are the normal projections of nodal acceleration, deformation rate, and imposed traction, respectively. 


\subsection{Stresses definition}

In order to close the formulation, the stresses of the internal force term (Eq.(24)) must be defined. In this method, stresses and strains are defined nodally and not at the Gauss points, as in the standard elemental FEM. Concerning the constitutive models, fluids are assumed to be incompressible and Newtonian, whereas a hypoelastic law is considered for the solid parts of the computational domain.

\subsubsection{Fluid nodal stress tensor}

For fluids, the nodal Cauchy stress tensor at ${ }^{n+1} t$ is computed according to the standard Newtonian model as

${ }^{n+1} \overline{\boldsymbol{\sigma}}=2 \mu_{f}\left({ }^{n+1} \overline{\boldsymbol{d}}-\frac{1}{3}{ }^{n+1} \bar{d}_{v} \boldsymbol{I}\right)+{ }^{n+1} \bar{p} \boldsymbol{I}$

where $\mu_{f}$ is the fluid dynamic viscosity. The nodal deformation rate tensor $\overline{\boldsymbol{d}}$ is obtained from Eq.(21) and the volumetric deformation rate from Eq.(20).

The fluid pressures are computed using the stabilized form of the continuity equation (Eq.(28))

Note that all terms of Eq.(35) are defined nodally. Thus, interpolation procedures are not required for the computation of the stresses. This represents an important advantage of nodal integration methods with respect to the elemental ones.

\subsubsection{Solid nodal stress tensor}

For solids, the nodal Cauchy stress tensor is computed according to the following hypoelastic model

$$
\begin{aligned}
{ }^{n+1} \overline{\boldsymbol{\sigma}}= & { }^{n} \overline{\boldsymbol{\sigma}}+2 \mu_{s} \Delta t\left({ }^{n+1} \overline{\boldsymbol{d}}-\frac{1}{3}{ }^{n+1} \bar{d}_{v} \boldsymbol{I}\right) \\
& +{ }^{n+1} \Delta \bar{p} \boldsymbol{I}
\end{aligned}
$$

where $\mu_{s}$ is the second Lamé constant.

For compressible solids, the nodal pressure variation $^{n+1} \Delta \bar{p}$ can be computed directly from the non-stabilized continuity equation (Eq.(26)) as

$$
{ }^{n+1} \Delta \bar{p}={ }^{n+1} \bar{p}-{ }^{n} \bar{p}=\kappa \Delta t^{n+1} \bar{d}_{v}
$$

Note that this direct relationship between the pressure and the volumetric strain rate is fulfilled exactly in a nodal integration framework because both variables are defined at the nodes $\left(\bar{d}_{v}\right.$ is computed nodally from the nodal velocities using Eq.(20)). This is in contrast to standard Gaussian integration methods where, typically, the volumetric strain measure is computed at the Gauss point while the pressures and the velocities are defined nodally. Combining Eq.(36) and Eq.(37), it yields:

$$
\begin{aligned}
{ }^{n+1} \overline{\boldsymbol{\sigma}}= & { }^{n} \overline{\boldsymbol{\sigma}}+2 \mu_{s} \Delta t\left({ }^{n+1} \overline{\boldsymbol{d}}-\frac{1}{3}{ }^{n+1} \bar{d}_{v} \boldsymbol{I}\right) \\
& +\kappa_{s} \Delta t^{n+1} \bar{d}_{v} \boldsymbol{I}
\end{aligned}
$$

and

${ }^{n+1} \overline{\boldsymbol{\sigma}}={ }^{n} \overline{\boldsymbol{\sigma}}+2 \mu_{s} \Delta t^{n+1} \overline{\boldsymbol{d}}+\lambda_{s} \Delta t^{n+1} \bar{d}_{v} \boldsymbol{I}$

where $\lambda_{s}$ is the first Lamé constant and $\kappa_{s}=$ $\lambda_{s}+\frac{2}{3} \mu_{s}$ is the solid bulk modulus.

Remarkably, in both Eqs.(38)-(39), which are equivalent to Eq.(36), the stresses are obtained using only the nodal velocities and not the nodal pressures. Hence, for the solid solution that does not need stabilization, the pressure is not used as nodal unknown because the velocity formulation is expected to have the same accuracy in stresses than the mixed velocity-pressure method. This also allows avoiding the duplication of the pressure degrees of freedom at the fluid-solid interface, as it was required in the elemental version of this FSI method [13], because nodal pressures are used as unknowns only for the fluid solution.

\section{PFEM remesh and fluid-solid interface detection}

In this approach, the free-surface fluid flow dynamics is described through the PFEM. This method combines the treatment of mesh nodes 
as material particles with an efficient remeshing technique to solve accurately large deformation problems with a Lagrangian FEM. Several examples of its successful application to complex non-linear problems can be found in the literature, e.g. $[30,4,36,25,6,11]$, just to mention some of the most recently published PFEM-based formulations.

In the PFEM, the FEM mesh needs to be rebuilt whenever it becomes excessively distorted. The new mesh is built over the cloud of points formed by the nodes of the previous (distorted) fluid mesh and all the solid boundaries (Figure 2b). This step implies the elimination of all the elements of the previous fluid mesh. Note that this step does not affect the solution of the proposed nodal integration method, as all the information is stored at nodes position, whereas it is critical for elemental integration methods that store historical variables at the Gauss points. The Delaunay Triangulation is then performed over the cloud of nodes (Figure 2c), and, finally, the Alpha Shape method is used to recognize the actual contours of the computational domain (Figure 2d).

The PFEM remeshing algorithm allows for the automatic detection of the fluid-solid interface. This occurs when fluid and solid domains are close enough to have at least one connecting element sufficiently small and regular to fulfill the Alpha Shape criteria (Figure 2d).

The conforming-mesh contact given by the PFEM remesh algorithm facilitates the FSI solution, as it is only required to assemble properly fluid and solid contributions into the solving linear system, as in a standard finite element analysis. On the other hand, it must be guaranteed that, at the interface, fluid and solid meshes have similar size and so avoiding topological inconveniences, such as the penetration of fluid nodes into the solid domain.

\section{Solution scheme}

The fluid-structure interaction problem is solved following the scheme of the so-called Unified (a) Mesh after computation of previous step

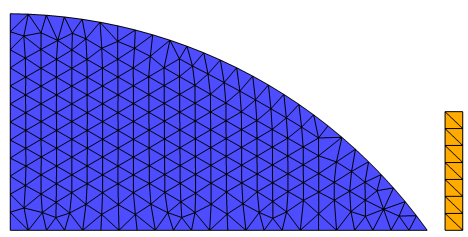

(b) Step 1: erase fluid elements

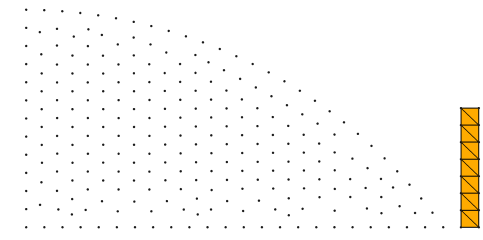

(c) Step 2: create Delaunay triangulation

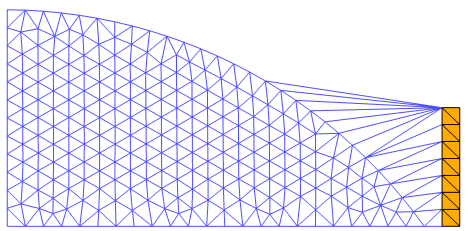

(d) Step 3: do Alpha Shape check

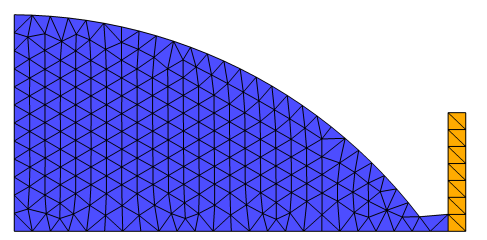

Fig. 2: PFEM remeshing and contact detection algorithm.

Formulation [15, 13]. Fluids and solids are solved within the same linear system in a monolithic way, guaranteeing automatically a strong FSI coupling and avoiding the iterations of fluid and solid solutions, as for staggered approaches. Furthermore, as nodal pressures are useless for the solid solution accuracy (see Section 2.5.2), it is not required to duplicate the pressure unknowns at the fluid-solid frontier, contrary to the homologous elemental approaches for FSI $[15,13]$.

Following previous works $[13,14,10]$, the nonlinear solution is obtained via a two-step iterative procedure. At each non-linear iteration, first, the linear momentum equations (Eq.(23)) are solved for the increments of nodal veloci- 
ties for both solid and fluid parts in monolithic FSI spirit. Then, in the updated configuration, the stabilized continuity equation (Eq.(28)) is computed only for fluid nodes. This scheme is repeated until the convergence of velocity and pressure solutions is not reached.

For a generic non-linear iteration $k+1$, the increments of nodal velocity are obtained from the following linearized form of the momentum equations as

$$
\left({ }^{k} \boldsymbol{M}+{ }^{k} \boldsymbol{K}\right){ }^{k+1} \boldsymbol{\Delta} \overline{\boldsymbol{v}}=-{ }^{k} \boldsymbol{R}_{m}
$$

where $\boldsymbol{\Delta} \overline{\boldsymbol{v}}$ is the vector of increments of nodal velocities, ${ }^{k} \boldsymbol{R}_{m}$ is the residual of the linear momentum balance at the previous non-linear iteration (Eq.(23)).

The nodal contribution of the mass matrix $M$ is computed as

$$
{ }^{k} M_{i i}^{I}=\frac{\rho}{2 \Delta t}{ }^{k} \Omega^{I}
$$

On the other hand, the stiffness matrix $\boldsymbol{K}$ is assembled nodally as [7]

${ }^{k} \boldsymbol{K}^{I}=\left[{ }^{k} \overline{\boldsymbol{B}}^{I}\right]^{T} \boldsymbol{C}{ }^{k} \overline{\boldsymbol{B}}^{I}{ }^{k} \Omega^{I}$

where matrix ${ }^{k} \overline{\boldsymbol{B}}^{I}$ is computed at each nonlinear iteration $k$ from Eq.(16).

The constitutive matrix $C$ can be written in a unified manner for fluids and solids as

$\boldsymbol{C}=\left[\begin{array}{ccc}\bar{\kappa} \Delta t+\frac{4 \bar{\mu}}{3} \bar{\kappa} \Delta t-\frac{2 \bar{\mu}}{3} & 0 \\ \bar{\kappa} \Delta t-\frac{2 \bar{\mu}}{3} \bar{\kappa} \Delta t+\frac{4 \bar{\mu}}{3} & 0 \\ 0 & 0 & \bar{\mu}\end{array}\right]$

where for solids $\bar{\mu}=\mu_{s} \Delta t$ and $\bar{\kappa}=\kappa_{s}$, while for fluids $\bar{\mu}=\mu_{f}$ and $\bar{\kappa}=\theta \kappa_{f}$. The parameter $\theta$ is used to reduce the fluid bulk modulus in order to avoid the ill-conditioning of the algebraic linear system. The procedure to set the optimum value of $\theta$ has been described in [12].

As mentioned in the previous sections, the stabilized continuity equation (Eq.(28)) is solved for fluids only. At each non-linear iteration, the new pressure increments $\left({ }^{k+1} \boldsymbol{\Delta} \bar{p}\right)$ are computed as

$$
\left(\boldsymbol{M}_{\kappa}+\boldsymbol{L}+\boldsymbol{M}_{\Gamma}\right)^{k+1} \boldsymbol{\Delta} \overline{\boldsymbol{p}}=-{ }^{k} \boldsymbol{R}_{\boldsymbol{c}}
$$

where each nodal component of matrix $\boldsymbol{M}_{\kappa}$ is computed as

$M_{\kappa}^{I}={\frac{1}{\kappa_{f} \Delta t}}^{k} \Omega^{I}$

being $\kappa_{f}$ the fluid actual bulk modulus is used.

For a generic time step $\left[{ }^{n} t ;^{n+1} t\right]$ of duration $\Delta t$, the solution scheme is summarized in the pseudo-code given in Algorithm 1.

\section{Validation tests}

In this section, five numerical tests are analyzed to validate the FSI scheme proposed in this article. The first two tests are proposed to validate the solid and the fluid nodal formulations, separately. In the third test, the interaction between a falling highly stiff object with a free-surface fluid is analyzed. Finally, two complex benchmarks tests involving huge fluid splashes and large displacements of solid structures are reproduced numerically and compared to the results of the literature.

\subsection{Dynamic response of a plane strain cantilever}

In this first numerical example, the dynamic response of the plane strain elastic cantilever presented in [1] is analyzed. The cantilever, illustrated in Figure 3, has length $L=25$ and height $D=4$. Young modulus, Poisson ratio and density are $E=10^{4}, \nu=0.25$ and $\rho=0.25$, respectively. At its free edge a load $P=40$ is applied.

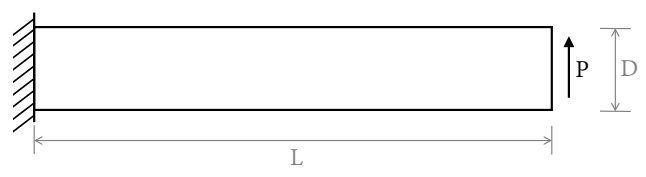

Fig. 3: Initial geometry of the plane strain cantilever.

The time evolution of the obtained vertical displacement of the top right corner is plotted in Figure 4 and compared to the results of 


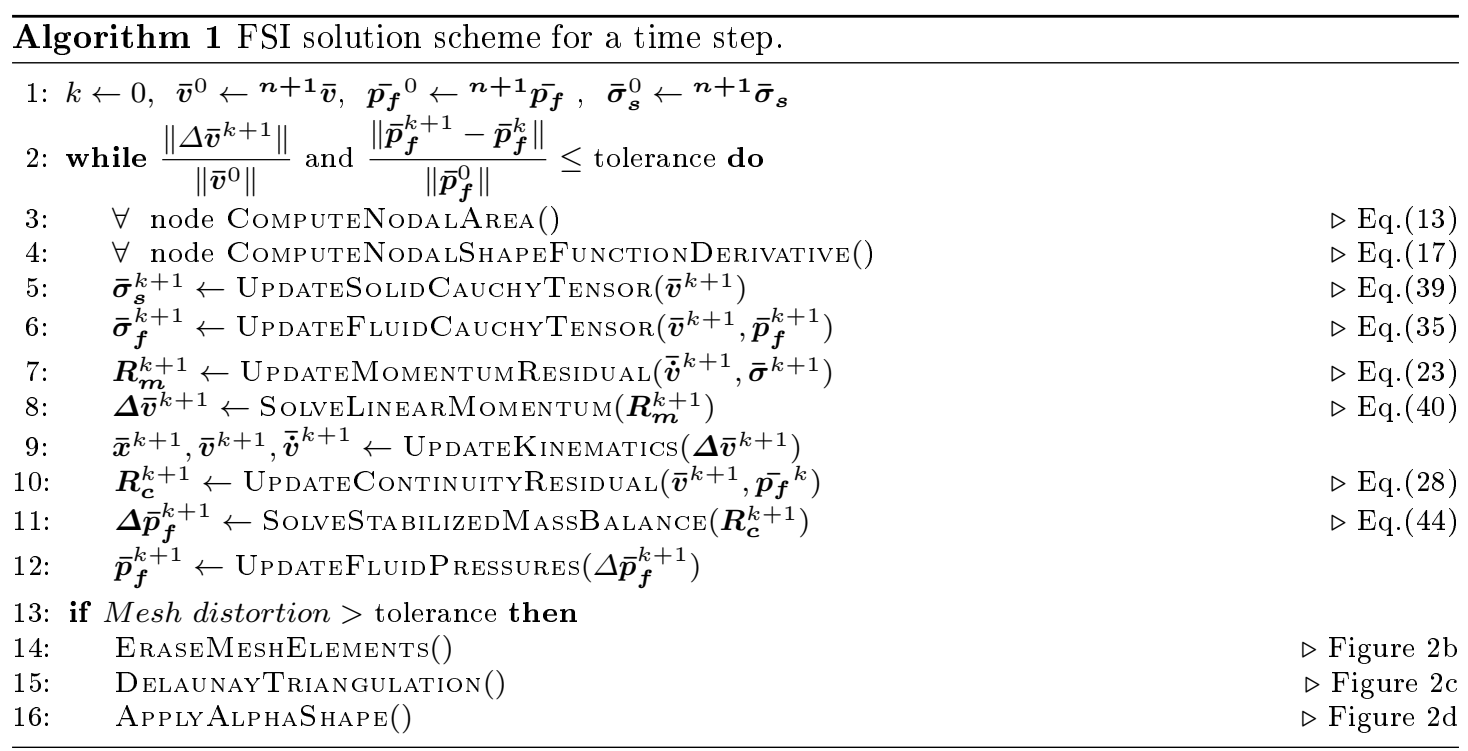

[1]. A very good agreeement with the reference solution is obtained.

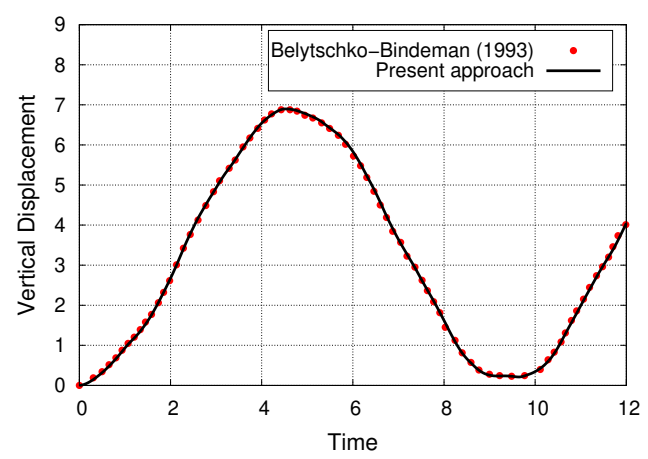

Fig. 4: Dynamic response of a plane strain cantilever. Time evolution of the top left corner vertical displacement. Reference solution: [1].

Figure 5 shows the cantilever at $t=4.55 \mathrm{~s}$, when the maximum vertical displacement is reached. The value of stress component $\sigma_{x}$ obtained at each node of the mesh is plotted over the deformed configuration.

It is interesting to analyze the convergence behavior of the proposed nodal approach and to compare it to the one obtained with a standard formulation with elemental integration. Seven different discretizations with mean ele-

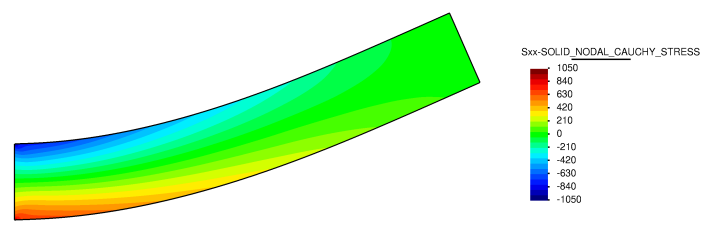

Fig. 5: Dynamic response of a plane strain cantilever. Results at $t=4.55 \mathrm{~s}$. The plotted contours are the nodal values of $\sigma_{x}$.

ment size spanning from 4 (one element per height) to 0.06125 (64 elements over the cantilever height), are used for the convergence analysis.

Figure 6a plots the maximum vertical displacement obtained for each mesh with the proposed nodal formulation, the hypoelastic one-field V-Element (velocity formulation with elemental integration [14]) and the two-field VP-Element (mixed velocity-pressure formulation with elemental integration [14]). The reference solution [1] is also plotted in the same graph. The results show that both elemental and nodal formulations converge to the expected results. However, as expected [21,20], while elemental FEM formulations converge to the solution from below (the computed solution is stiffer than the expected one), the nodal integration method gives an upper bound solu- 
tion (the computed solution is softer than the expected one).

(a)

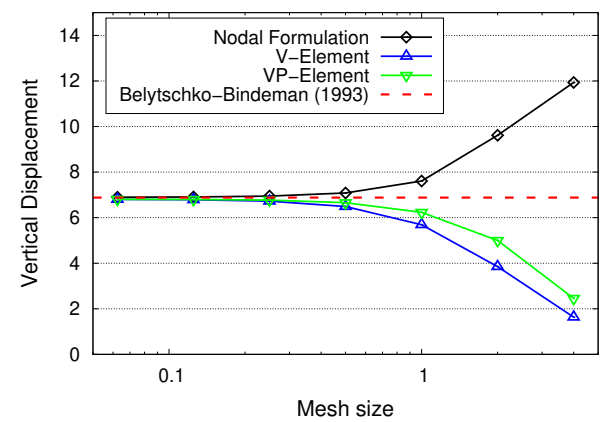

(b)

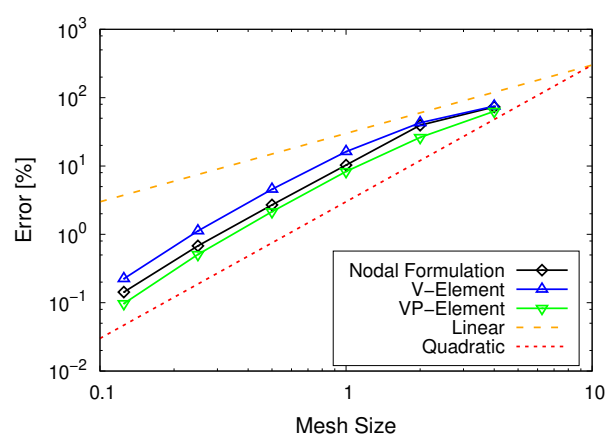

Fig. 6: Dynamic response of a plane strain cantilever. Maximum vertical displacement and percentage error for different meshes. Reference solution: [1]. V-Element and VP-Element are Velocity and Velocity-Pressure formulations with elemental integration [14].

An error measure is defined to compare quantitatively the results of the proposed nodal model to those of the two strategies with elemental integration. For the three methods, the error of each mesh is computed as the percentage of the difference between its solution and the one obtained with the finest mesh, normalized by the expected value of [1] (6.88). The results plotted in Figure show that the three formulations give a quadratic convergence with this error measure. However, for the same mesh, the nodal approach (one-field method) gives a more accurate solution versus its elemental counterpart (the V-Element), and a slightly higher error than the mixed VP-Element.

In summary, these results confirm that in solid mechanics, homologous nodal and elemental formulations can be used together to bound the expected solution. Furthermore, they show that, for the same mesh, the nodal approach gives a more accurate solution than the corresponding one-field elemental method. This is attributable to the fact that, by construction, in the nodal FEM, the mesh nodes have larger support than in the elemental FEM. In other words, in a nodal model, there is a wider coupling between the degrees of freedom of the entire mesh [7]. This translates in a larger bandwidth of the stiffness matrix, which in turn yields to a higher computational cost to build and solve the linear system, but also to higher accuracy of the method. On the other hand, the one-field nodal formulation has shown to give a slightly higher error than the two-field velocity-pressure elemental method. Note that this result is in line with what was found in [10] for fluid dynamics problems.

\subsection{Water dam break against a rigid wall}

This second test is used to validate the fluid counterpart of the proposed FSI method. The problem consists of reproducing the collapse of a water column against a rigid wall presented in [22]. The initial geometry of the test is given in Figure 7 . The fluid properties are: density $\rho=997 \mathrm{~kg} / \mathrm{m}^{3}$, dynamic viscosity $\mu=$ $0.00089 \mathrm{~Pa} \cdot \mathrm{s}$ and bulk modulus $\kappa=2.1 \cdot 10^{9} \mathrm{~Pa}$. A finite element mesh of 23750 triangles with mean size $3 \mathrm{~mm}$ has been used.

Figure 8 shows a qualitative comparison between the numerical results and the experimental observations. The pictures show that the proposed nodal PFEM is capable to reproduce the complex dynamics of the experimental test, from the first collapse of the water column to its impact against the terminal rigid wall and consequent waves and splashes formation. 


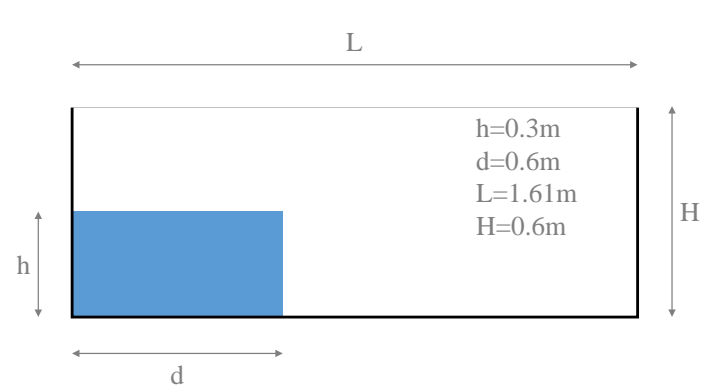

Fig. 7: Water dam break against a rigid wall. Initial geometry.

In Figure 9 the dimensionless time $\left(t^{*}\right)$ evolution of normalized residual height $\left(h^{*}\right)$ and wave front position $\left(x^{*}\right)$ is plotted. The dimensionless variables are computed as $t^{*}=$ $\sqrt{t /(g / h)}, h^{*}=y_{\max } / h$ and $x^{*}=\left(x_{\max }-\right.$ d) $/ d$.

The plotted results show a very good agreement between the proposed numerical method and the laboratory test.

The accuracy of the numerical simulation is also confirmed by the analysis of the pressure exerted by the fluid on the rigid wall. In [22], three pressure sensors were placed at different heights of the rigid wall, namely, sensor $A$ at $y=0.03 \mathrm{~m}$, sensor $B$ at $y=0.05 \mathrm{~m}$ and sensor $C$ at $y=0.08 \mathrm{~m}$. In Figure 10, the recorded time evolution of the pressure values at the sensors is plotted together with the numerical results obtained with the proposed nodal formulation. The plotted dimensionless pressure is computed as $p^{*}=p /(g \rho h)$. The results show again the very good agreement between the numerical and the experimental results. The proposed method is capable to capture accurately the time of the impact of the fluid stream against the sensors and, despite some reduced oscillations, the time evolution of the exerted fluid pressure.

\subsection{Wedge water entry}

In this section, the entry of a solid wedge into a basin filled with water is reproduced numerically with the proposed nodal-based formula- (a) $t=0.160 \mathrm{~s}$

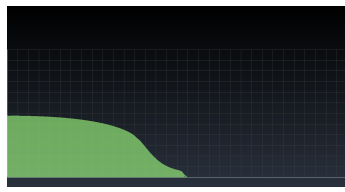

(b) $t=0.277 \mathrm{~s}$

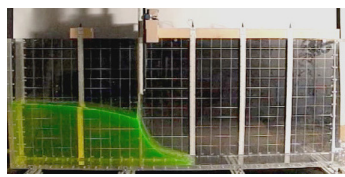

(c) $t=0.450 \mathrm{~s}$

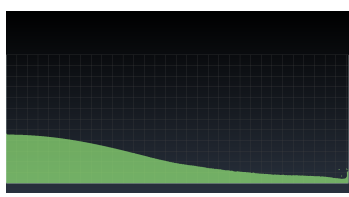

(d) $t=0.862 \mathrm{~s}$

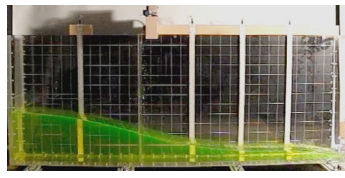

(e) $t=1.023 \mathrm{~s}$

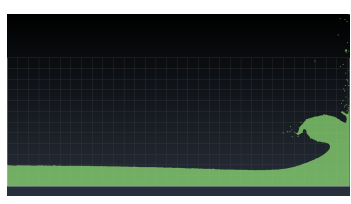

(f) $t=1.167 \mathrm{~s}$
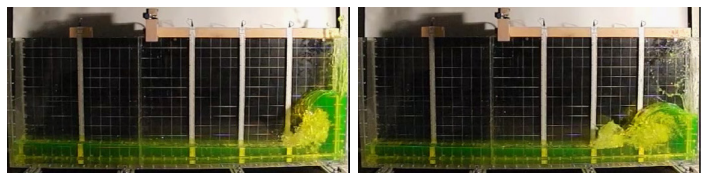

Fig. 8: Water dam break against a rigid wall. Results of the proposed nodal PFEM results (upper figures) and experimental observations [22] (lower figures) at six time instants.

tion for FSI. The problem is the two-dimensional adaptation of the experimental test presented in [33]. The wedge is made falling on the water at rest from a heigh of $1.3 \mathrm{~m}$. The initial geometry of the test is illustrated in Figure 11.

The wedge is modeled as a hypoleastic solid with high stiffness. In particular, the wedge's material properties are $E=10^{9} \mathrm{~Pa}, \nu=0$ and 
(a)

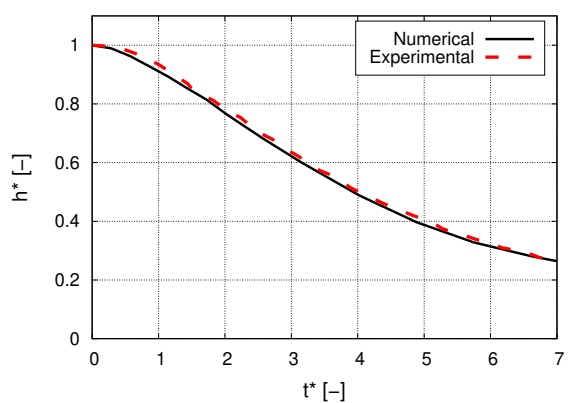

(b)

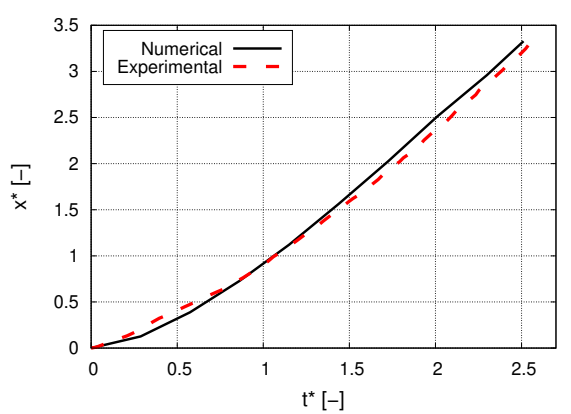

Fig. 9: Water dam break against a rigid wall. Dimensionless time $\left(t^{*}=\sqrt{t /(g / h)}\right)$ evolution of dimensionless residual height $\left(h^{*}=y_{\max } / h\right)$ and wave front position $\left(x^{*}=\left(x_{\max }-d\right) / d\right)$. Experimental results from [22].

$\rho_{s}=466.07 \mathrm{~kg} / \mathrm{m}^{3}$, while for the water $\rho_{f}=$ $10^{3} \mathrm{~kg} / \mathrm{m}^{3}, \mu_{f}=10^{-3} \mathrm{~Pa} \cdot \mathrm{s}$ and $\kappa_{f}=2.1$. $10^{9} \mathrm{~Pa}$ have been considered.

In this test, it is crucial to capture accurately the actual time of the wedge's impact on the water. Therefore, a very fine mesh is required to reduce the size of the PFEM contact elements (see Figure 2). Different meshes have been analyzed to select the optimum mesh size for this problem. Figure 12 shows the time evolution of the vertical velocity of the wedge (taken at the lower vertex of the wedge) obtained with the different meshes, whose mean size span from $6 \mathrm{~cm}$ to $0.75 \mathrm{~cm}$. The plot shows converged results for the finest mesh. Hence, the test is analyzed using the results obtained with the discretization with a mean mesh size (a) Sensor $A$

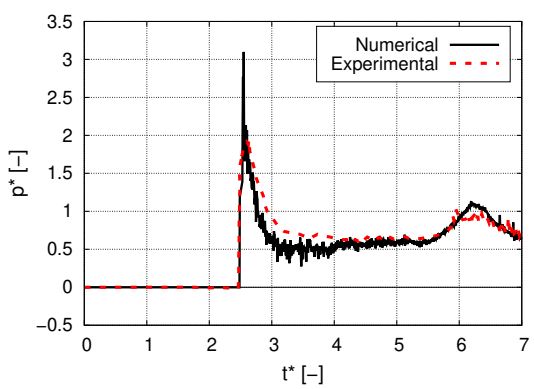

(b) Sensor $B$

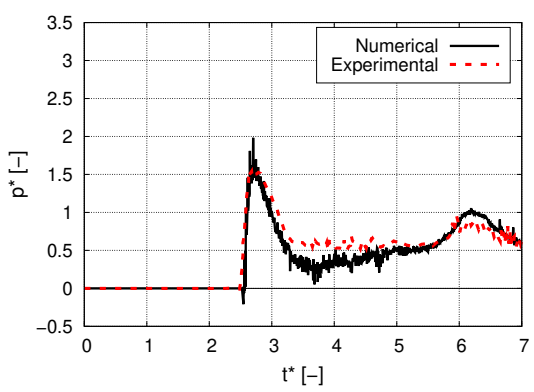

(c) Sensor $C$

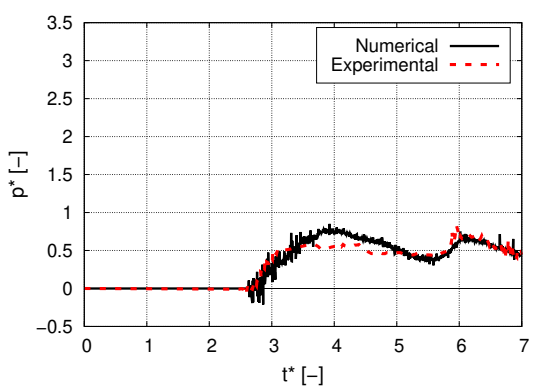

Fig. 10: Water dam break against a rigid wall. Dimensionless time $\left(t^{*}=\sqrt{t /(g / h)}\right)$ evolution of normalized pressure $\left(p^{*}=p /(g \rho h)\right)$ at three different positions of the terminal vertical wall (positions $[x, y]$ of sensors A, B and C are $[1.61 m, 0.03 m]$, $[1.61 \mathrm{~m}, 0.05 \mathrm{~m}]$ and $[1.61 \mathrm{~m}, 0.08 \mathrm{~m}]$, respectively). Experimental results from [22].

of $0.75 \mathrm{~cm}$ and composed by 6800 and 112150 solid and fluid triangular elements, respectively.

In Figure 13 the numerical results at five time instants are plotted. After a free-fall regime, the wedge after a free fall impacts the water 


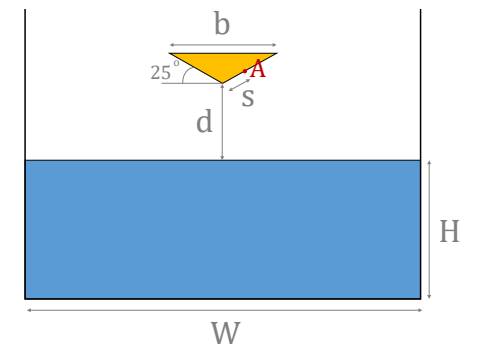

Fig. 11: Wedge water entry. Initial geometry.

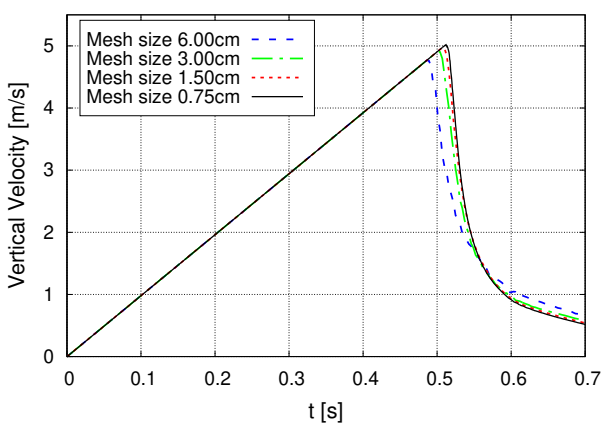

Fig. 12: Wedge water entry. Time evolution of the vertical velocity of the wedge obtained for different meshes.

reservoir. This occurs at around $t=0.514 \mathrm{~s}$ when the edge reached a vertical velocity of around $5 \mathrm{~m} / \mathrm{s}$ (Figure 13a). Then, the wedge penetrates the fluid generating two symmetrical waves at its borders while it is decelerating.

In Figure 14, the time evolution of the vertical velocity and the pressure computed at point A of Figure 11 are compared to the ones obtained experimentally in [33] and numerically in [31]. The time $\hat{t}=0$ used in the graphs of Figure 14 represent the moment of the impact of the wedge on the water reservoir $(\hat{t}=$ $\left.t-t_{\text {impact }}=t-0.514\right)$

Once again, the results obtained with the proposed nodal PFEM for FSI show a very good agreement with the results of the literature. (a) $\mathrm{t}=0.514 \mathrm{~s}$

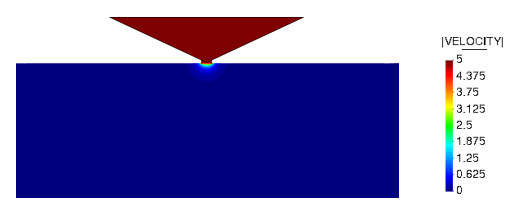

(b) $\mathrm{t}=0.555 \mathrm{~s}$

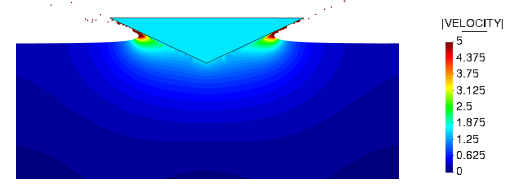

(c) $\mathrm{t}=0.600 \mathrm{~s}$

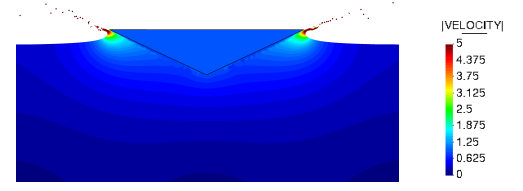

(d) $\mathrm{t}=0.650 \mathrm{~s}$

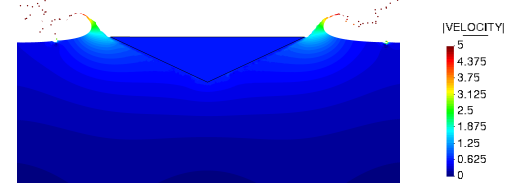

(e) $\mathrm{t}=0.700 \mathrm{~s}$

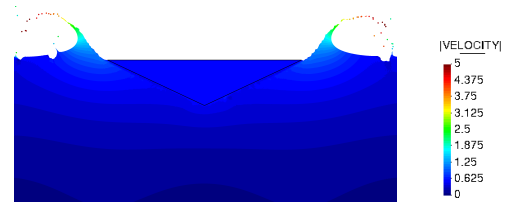

Fig. 13: Wedge water entry. Numerical results at five time instants.

5.4 Filling of an elastic container with a viscous fluid

This problem is a benchmark test for free-surface fluid interacting with highly deformable solids. The test was initially proposed in [5] and then slightly modified in [13] to improve its reproducibility. The two-dimensional test consists of the sudden release of a mass of a highly vis- 
(a)

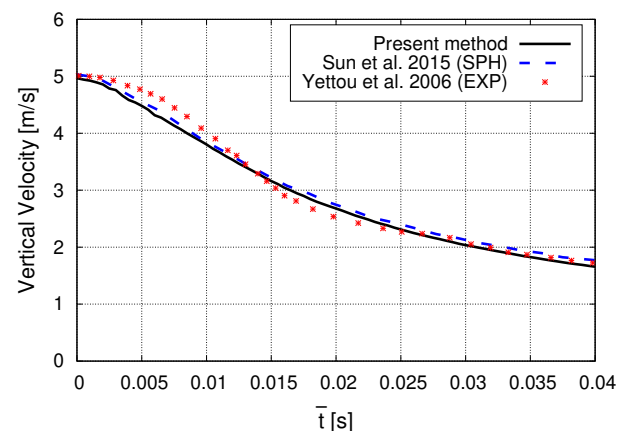

(b)

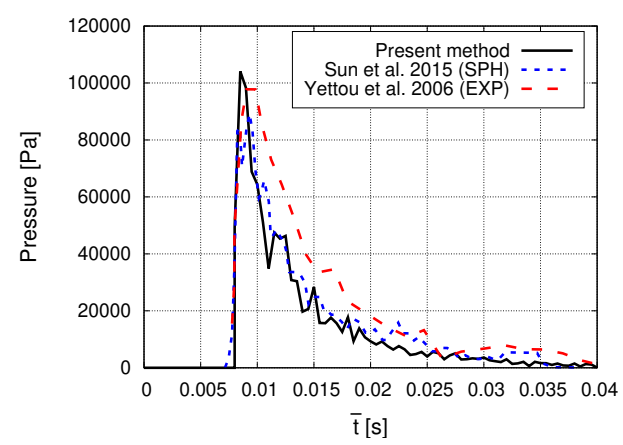

Fig. 14: Wedge water entry. (a) Time evolution of the vertical velocity of the wedge. (b) Time evolution of the pressure at the point $A$ of Figure 11. Reference results: SPH formulation [31] and experimental test [33].

cous fluid $\left(\rho_{f}=10^{3} \mathrm{~kg} / \mathrm{m}^{3}, \mu_{f}=10^{2} \mathrm{~Pa} \cdot \mathrm{s}\right.$ and $\kappa_{f}=2.1 \cdot 10^{9} \mathrm{~Pa}$ ) over an elastic U-shaped container $E=2.1 \cdot 10^{7} \mathrm{~Pa}, \nu=0.3$ and $\rho_{s}=$ $20 \mathrm{~kg} / \mathrm{m}^{3}$ ). The initial geometry of the problem is provided in Figure 15.

A mean mesh size of $3 \mathrm{~cm}$ is used. The initial mesh of the viscous fluid is formed by 19530 triangular elements, while the one of the elastic container by 7507 ones.

The works taken as reference for this test are the recent publications [4] and [23], where two staggered methods for FSI were presented. Both methods use a PFEM with elemental integration for the fluid solution and a FEM software (a commercial one in [23] and an in-house code in [4]) for the solid solution.

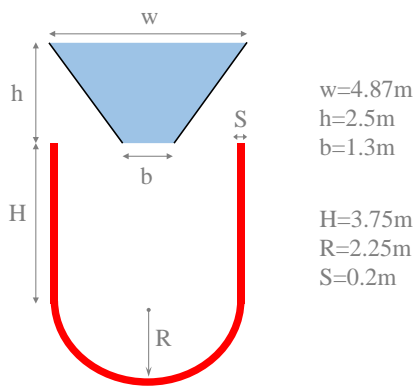

Fig. 15: Filling of an elastic container with a viscous fluid.

A qualitative comparison between the results obtained with the proposed nodal FSI scheme (pictures at the left) and those presented in [4] (pictures at the right) is shown in Figure 16 for six representative time instants. Despite the complexness of the problem, the figure shows a very good agreement between the two different PFEM formulations.

A quantitative comparison with the literature results is given in Figure 17 that reports the time evolution of the vertical displacement of the bottom of the elastic structure obtained by the two reference works ([4] and [23]) and the proposed nodal PFEM. The three formulations show a very good agreement, especially during the first $4 s$ of analysis when all the peaks of vertical displacement are well captured by all methods. After this first $4 \mathrm{~s}$ of analysis, the three formulations show some discrepancies, but this is almost unavoidable in such unsteady problems. However, it is worth noting that the three solutions oscillate around almost the same value of vertical displacement.

In this test, it is particularly important to guarantee the mass conservation of the fluid domain. Indeed, changes in the fluid mass lead to variations of the load exerted over the elastic container and, thus, to different displacement field results. Moreover, it is well known that mass conservation is one of the most critical aspects of a PFEM analysis [9], above all when highly unsteady problems are considered. For these two reasons, the mass variation has 
(a) $\mathrm{t}=1.04 \mathrm{~s}$

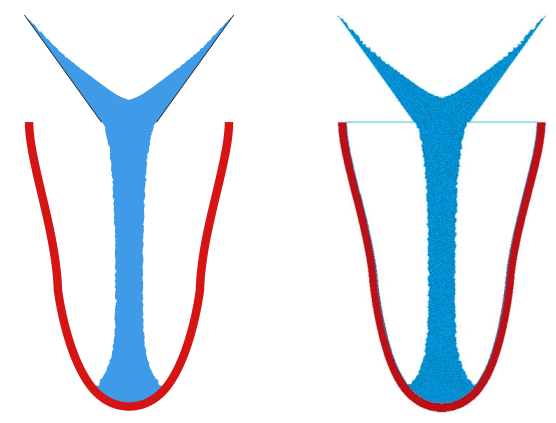

(c) $\mathrm{t}=2.0 \mathrm{~s}$

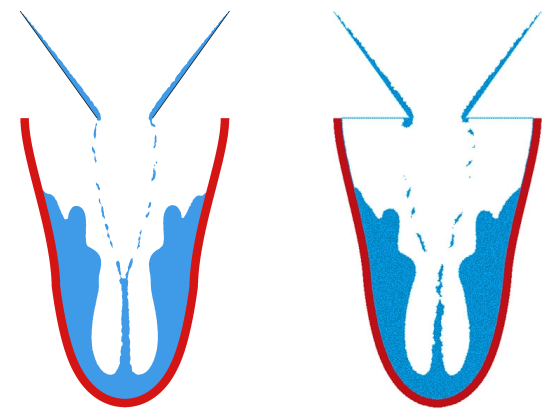

(e) $\mathrm{t}=3.5 \mathrm{~s}$
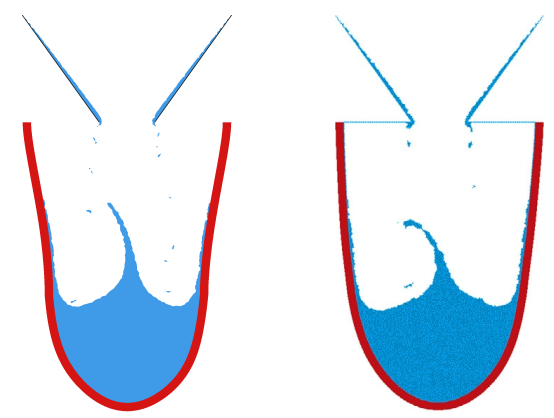

(b) $\mathrm{t}=1.5 \mathrm{~s}$

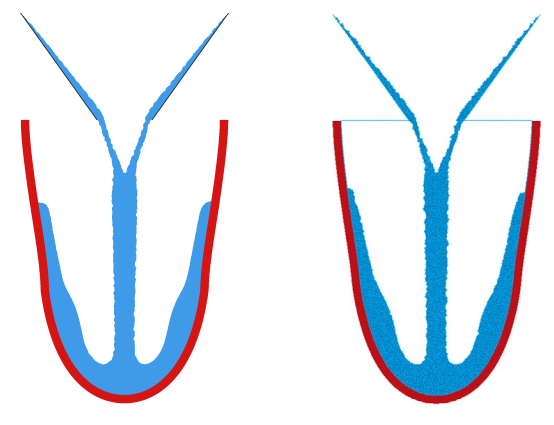

(d) $\mathrm{t}=2.8 \mathrm{~s}$

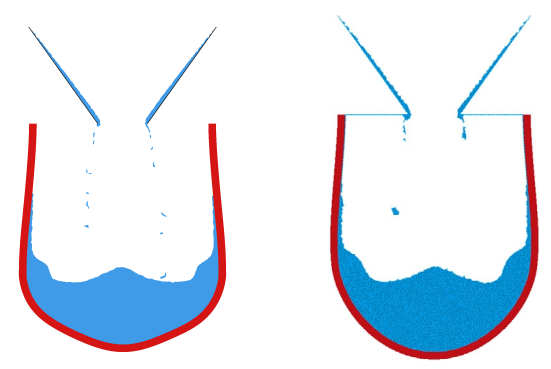

(f) $\mathrm{t}=10.0 \mathrm{~s}$

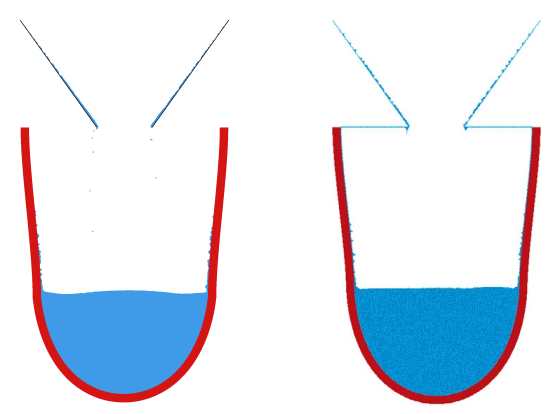

Fig. 16: Filling of an elastic container with a viscous fluid. Results at six time instants. For each sub-figure, the results plotted at the left hand side are obtained with the proposed approach, while those of the right are from [4].

been monitored in this test and its time evolution is reported in Figure 18. The graph shows that the overall mass variation keeps limited (less than 1.5\%) for all the duration of the analysis. This is remarkable considering the unsteadiness of the fluid flow and shows the good mass conservation skills of the used nodal PFEM.

\subsection{Collapse of water column against an} elastic structure

The collapse of a water column against a deformable membrane is a well-known benchmark problem for FSI involving free-surface fluids [32]. The initial geometry of the test is provided in Figure 19. For the water, a density $\rho=$ $1000 \mathrm{~kg} / \mathrm{m}^{3}$ and a viscosity $\mu=0.001 \mathrm{~Pa} \cdot \mathrm{s}$ have been used. The elastic membrane has density 


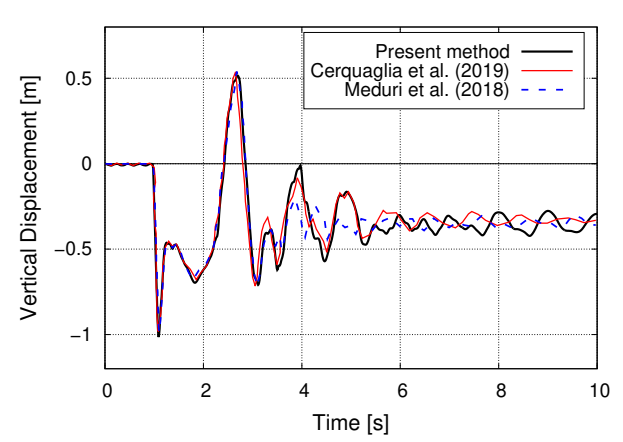

Fig. 17: Filling of an elastic container with a viscous fluid. Time evolution of the vertical displacement obtained at the bottom of the elastic structure. Reference results from [4] and [23].

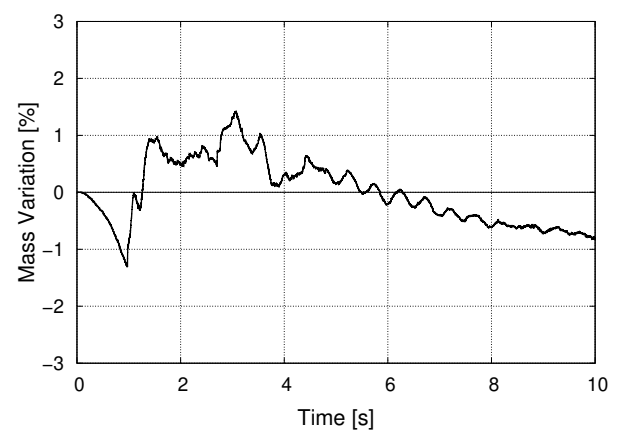

Fig. 18: Filling of an elastic container with a viscous fluid. Time evolution of the fluid mass variation with respect to the initial mass.

$\rho=2500 \mathrm{~kg} / \mathrm{m}^{3}$, Young modulus $E=10^{3} \mathrm{KPa}$ and Poisson ratio $\nu=0$. A mesh with a mean

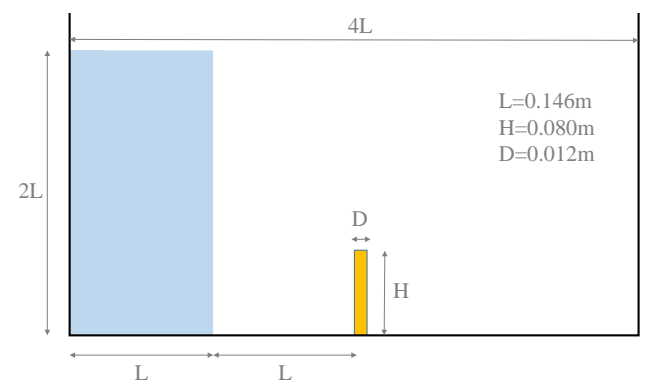

Fig. 19: Collapse water column against an elastic structure. Initial geometry.

mesh size of $2 \mathrm{~mm}$ has been used for both solid

and fluid computational domain. The initial solid mesh is composed of 540 triangular elements, while the fluid domain is initially discretized with 24375 triangles.

In Figure 20, some representative pictures of the test are given. The images show that the proposed method can capture the complex behavior of this unsteady problem, including the initial dam break, the large motion of the elastic membrane induced by the water impact force, and the huge water splashes forming after the impact to the retaining vertical wall.

(a) $t=0.25 \mathrm{~s}$

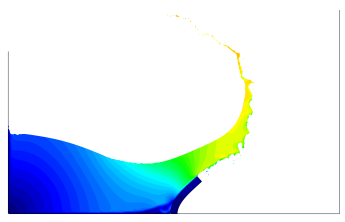

(c) $t=0.60 \mathrm{~s}$

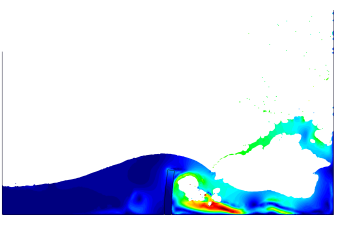

(e) $t=0.75 \mathrm{~s}$

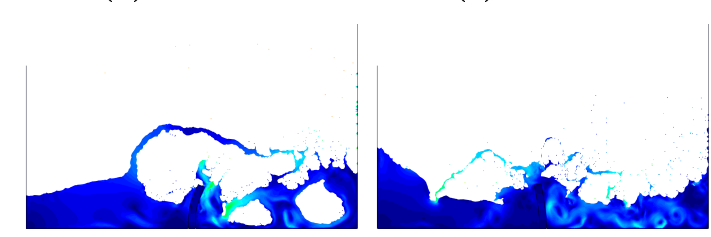

(g) $t=1.00 \mathrm{~s}$

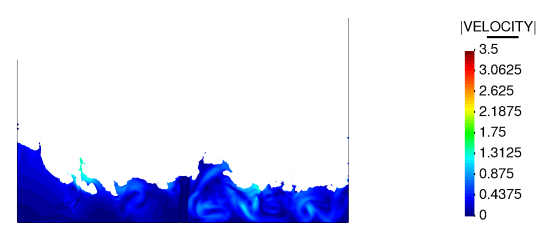

Fig. 20: Collapse water column against an elastic structure. Numerical results at different time instants. The velocity contours are plotted over the solid and the fluid meshes. 
Figure 21 plots the time evolution of the horizontal displacement of the left top corner of the structure obtained by the proposed method and those obtained by other FSI formulations of the literature. The results show that for the

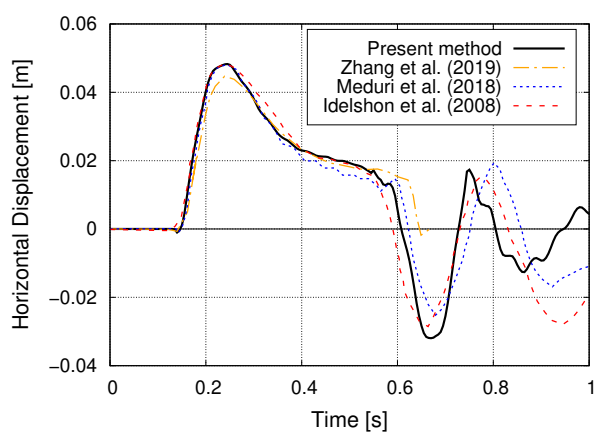

Fig. 21: Collapse water column against an elastic structure. Time evolution of the horizontal displacement of the left top corner of the structure. Results from the proposed method and other numerical strategies of the literature $([17,24,37])$.

first $0.5 \mathrm{~s}$ of analysis there is a good agreement between the different methods, while, after $0.5 s$, a certain discrepancy of results is observed. This is due to the high unsteadiness that characterizes the second part of this test. Furthermore, it must be considered that the dynamics of the test changes significantly with small variations on the prediction of the deflection of the elastic membrane. For example, a stiffer response of the structure yields a higher upwards motion of the fluid and a delayed return wave on the structure. Taken all this into account, the overall agreement with the literature results confirms again the validity of the proposed method for FSI problems.

Finally, it is interesting to check if, also in this FSI test, it is possible to bound the expected solution by combining the results of elemental and nodal approaches, as it has been done for the solid dynamics test of Section 5.1. As already explained, a nodal FEM is expected to give an upper bound solution, contrary to an elemental method that converges to the expected result from below. Exploit- ing this feature, one should be able to get a range of possible solutions using the results of nodal and elemental approaches, even if obtained from coarse meshes. For this purpose, the same dam-break problem is solved using a coarse discretization of mean size $6 \mathrm{~mm}$ (50 solid elements and 2700 fluid ones) with both the nodal and the elemental methods. Figure 22 shows the first 0.4 s of the time evolution of the maximum horizontal displacement of the structure obtained with the proposed nodal method and the elemental PFEM [13], using the coarse and the fine mesh. The graph shows that the expected solution (in this case, the one obtained with the finest mesh) is effectively bounded by the coarse mesh solutions of elemental (lower bound) and nodal (upper bound) methods. This confirms that, also for FSI problems, one could use homologous nodal and elemental methods to have an estimation of the problem solution from relatively coarse finite-element meshes. The extension and formalization of this property to more complex non-linear FSI analyses are left for future studies and considered out of the scope of this work.

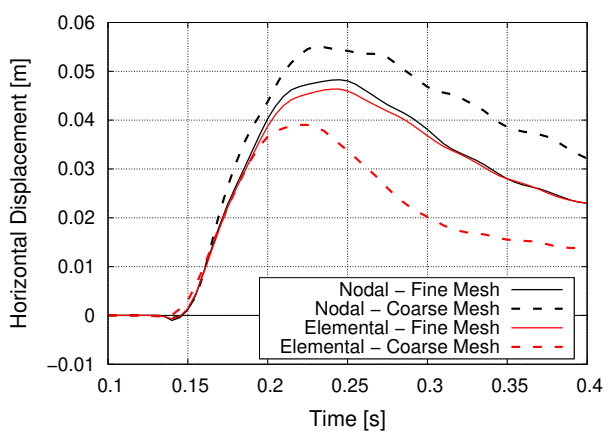

Fig. 22: Collapse water column against an elastic structure. Time evolution of the horizontal displacement of the left top corner of the structure obtained with the proposed nodal PFEM and the elemental PFEM [13], for a coarse and a fine mesh. 


\section{Concluding remarks}

This work presented a new nodally integrated fully Lagrangian method for Fluid-Structure Interaction (FSI) problems. The fluid parts of the computational domain are solved with a recently proposed Particle Finite Element Metho (PFEM) with nodal integration [10], whereas the solids are modeled with a novel hypoelastic model with nodal integration. The whole coupled problem is solved with a monolithic approach to guarantee a strong coupling to the FSI solution. It is worth remarking that the proposed method is one of the few examples of FEM formulations with nodal integration for FSI problems with free-surface fluids [39,38, 37], and the first one involving a PFEM formulation.

In FSI analyses involving compressible solids and incompressible fluids, the use of nodal integration has the advantage versus standard methods with Gaussian integration of avoiding the duplication of pressure degrees of freedom at the fluid-solid interface, as nodal pressures are used for the fluid solution only.

The proposed numerical method has been validated against several benchmark problems.

In the first test, the convergent behavior of the nodally-integrated solid model has been proved by analyzing the dynamic bending of an elastic cantilever. The test has been also used to show the characteristic property of nodalbased FEM of giving an upper bound solution to the elastic problem.

Then, the collapse of a water column against a rigid vertical wall has been studied. The numerical results have shown very good agreement with the experimental observations in terms of water motion and values of pressure exerted on the retaining walls.

In the third and fourth validation tests, the nodal algorithm has shown to be able to solve accurately FSI problems in presence of both stiff and soft elastic bodies and highly unsteady free-surface fluid flow. A very good agreement with laboratory experiments and nu- merical results of the literature has been found in all cases.

In the last test, it has been shown that, also for FSI problems, it is possible to bound the expected solution with the coarse-mesh results obtained with the nodally integrated method and its homologous FEM with Gaussian integration.

The accuracy of the obtained results proves the suitability of the proposed PFEM-FEM method with nodal integration for the solution of complex FSI problems involving large motions of the solid domains and huge changes of the topology of the free-surface fluid.

From a broader perspective, this work should be interpreted as a further step, after [10], towards the derivation of a unified PFEM formulation for large deformation solid and fluid bodies, including also phase change phenomena. To achieve this objective two further developments are required. First, the effects of PFEM remeshing on the solid solution must be carefully analyzed. Then, a unified constitutive model with a smooth phase transition has to be defined and implemented into the nodal integration framework. Both developments are left for future work.

Acknowledgements The Spanish Ministry of Economy and Competitiveness (Ministerio de Economia y Competitividad, MINECO) through the project PRECISE (BIA2017- 83805-R) is gratefully acknowledged by the author for the economic support.

\section{Conflict of Interest}

The author declares that he has no conflict of interest.

\section{References}

1. T. Belytschko and L.P. Bindeman. Assumed strain stabilization of eight node hexahedral element. Computer Methods In Applied Mechanics And Engineering, 105:225-260, 1993.

2. T. Belytschko, W.K. Liu, B. Moran, and K.I. Elkhodadry. Nonlinear Finite Elements For Continua And Structures. Second Edition. John Wiley \& Sons, New York, 2014. 
3. F. Brezzi. On the existence, uniqueness and approximation of saddle-point problems arising from lagrange multipliers. Revue française d'automatique, informatique, recherche opérationnelle. Série rouge. Analyse numérique, 8(R-2):129-151, 1974.

4. M.L. Cerquaglia, D. Thomas, R. Boman, V. Terrapon, and J.P. Ponthot. A fully partitioned lagrangian framework for fsi problems characterized by free surfaces, large solid deformations and displacements, and strong added-mass effects. Computer Methods in Applied Mechanics and Engineering, 348:409-442, 2019.

5. M. Cremonesi, A. Frangi, and U. Perego. A lagrangian finite element approach for the analysis of fluid-structure interaction problems. International Journal of Numerical Methods in Engineering, 84(5):610-630, 2010.

6. M. Cremonesi, S. Meduri, and U. Perego. Lagrangian-eulerian enforcement of nonhomogeneous boundary conditions in the particle finite element method. Computational Particle Mechanics, pages 1-16, 2019.

7. C.R. Dohrmann, M.W. Heinstein, J. Jung, S.W. Key, and W.R. Witkowski. Node-based uniform strain elements for three-node triangular and four-node tetrahedral meshes. International Journal of Numerical Methods in Engineering, 47(9):1549-1568, 2000.

8. H. Feng, X.Y. Cui, and G.Y. Li. A stable nodal integration method with strain gradient for static and dynamic analysis of solid mechanics. Engineering Analysis with Boundary Elements, 62:78-92, 2016.

9. A. Franci and M. Cremonesi. On the effect of standard PFEM remeshing on volume conservation in free-surface fluid flow problems. Computational Particle Mechanics, 4(3):331343, 2017.

10. A. Franci, M. Cremonesi, U. Perego, and E. Oñate. A lagrangian nodal integration method for free-surface fluid flows. Computer Methods in Applied Mechanics and Engineering, 361:112816, 2020.

11. A. Franci, I. de Pouplana, G. Casas, M.A. Celigueta, J. González-Usúa, and E. Oñate. PFEM-DEM for particle-laden flows with free surface. Computational Particle Mechanics, pages 1-20, 2019.

12. A. Franci, E. Oñate, and J.M. Carbonell. On the effect of the bulk tangent matrix in partitioned solution schemes for nearly incompressible fluids. International Journal for Numerical Methods in Engineering, 102 (3-4):257-277, 2015.

13. A. Franci, E. Oñate, and J.M. Carbonell. Unified lagrangian formulation for solid and fluid mechanics and fsi problems. Computer Meth- ods in Applied Mechanics and Engineering, 298:520-547, 2016.

14. A. Franci, E. Oñate, and J.M. Carbonell. Velocity-based formulations for standard and quasi-incompressible hypoelastic-plastic solids. International Journal for Numerical Methods in Engineering, 107 (11):970-990, 2016.

15. S.R. Idelsohn, J. Marti, A. Limache, and E. Oñate. Unified lagrangian formulation for elastic solids and incompressible fluids: Applications to fluid-structure interaction problems via the PFEM. Computer Methods In Applied Mechanics And Engineering, 197(19-20):17621776, 2008

16. S.R. Idelsohn, E. Oñate, and F. Del Pin. The particle finite element method: a powerful tool to solve incompressible flows with free-surfaces and breaking waves. International Journal for Numerical Methods in Engineering, 61(7):964989, 2004.

17. S.R. Idelsohn, E. Oñate, F. Del Pin, and N. Calvo. Fluid-structure interaction using the particle finite element method. Computer methods in applied mechanics and engineering, 195(17-18):2100-2113, 2006.

18. E. Li, Z. Zhang, C.C. Chang, G.R. Liu, and Q. Li. Numerical homogenization for incompressible materials using selective smoothed finite element methods. Composite Structures, 123:216-232, 2015.

19. G.R. Liu, T.T. Nguyen, K.Y. Dai, and K.Y. Lam. Theoretical aspects of the smoothed finite element method (sfem). International Journal of Numerical Methods in Engineering, 71(8):902-930, 2006.

20. G.R. Liu, T.T. Nguyen, H. Nguyen-Xuan, and K.Y. Lam. A node-based smoothed finite element method (ns-fem) for upper bound solutions to solid mechanics problems. Computers and Structures, 87:14-26, 2009.

21. G.R. Liu and G.Y. Zhang. Upper bound solution to elasticity problems: A unique property of linearly conforming point interpolation method (lc-pim). International Journal of $\mathrm{Nu}$ merical Methods in Engineering, 74:1128-1161, 2008.

22. L. Lobovský, E. Botia-Vera, F. Castellana, J. Mas-Soler, and A. Souto-Iglesias. Experimental investigation of dynamic pressure loads during dam break. Journal of Fluids and Structures, 48:407-434, 2014.

23. S. Meduri, M. Cremonesi, and U. Perego. An efficient runtime mesh smoothing technique for 3d explicit lagrangian free-surface fluid flow simulations. International Journal for Numerical Methods in Engineering, 117(4):430-452, 2019.

24. S. Meduri, M. Cremonesi, U. Perego, O. Bettinotti, A. Kurkchubasche, and V.M. Oancea. 
A partitioned fully explicit lagrangian finite element method for highly nonlinear fluidstructure interaction problems. International Journal for Numerical Methods in Engineering, 113:43-64, 2018.

25. L. Monforte, P. Navas, J.M. Carbonell, M. Arroyo, and A. Gens. Low-order stabilized finite element for the full biot formulation in soil mechanics at finite strain. International Journal for Numerical and Analytical Methods in Geomechanics, 43(7):1488-1515, 2019.

26. T. Nguyen-Thoi, G.R. Liu, and K.Y. Lam G.Y. Zhang. A face-based smoothed finite element method (fs-fem) for 3d linear and geometrically non-linear solid mechanics problems using 4-node tetrahedral elements. International Journal of Numerical Methods in Engineering, 78(3):324-353, 2009.

27. E. Oñate, A. Franci, and J.M. Carbonell. Lagrangian formulation for finite element analysis of quasi-incompressible fluids with reduced mass losses. International Journal for Numerical Methods in Fluids, 74(10):699-731, 2014.

28. E. Oñate, S.R. Idelsohn, F. Del Pin, and R. Aubry. The particle finite element method. an overview. International Journal for Computational Methods, 1:267-307, 2004

29. P. Ryzhakov, E. Oñate, and S.R. Idelsohn. Improving mass conservation in simulation of incompressible flows. International Journal of Numerical Methods in Engineering, 90:1435$1451,2012$.

30. F. Salazar, J. San-Mauro, M.A. Celigueta, and E. Oñate. Shockwaves in spillways with the particle finite element method. Computational Particle Mechanics, pages 1-13, 2019.

31. P. Sun, F. Ming, and A. Zhang. Numerical simulation of interactions between free surface and rigid body using a robust sph method. Ocean Engineering, 98:32-49, 2015.

32. E. Walhorn, A. Kolke, B. Hubner, and D.Dinkler. Fluid-structure coupling within a monolithic model involving free surface flows. Computer \& Structures Methods in Applied Mechanics and Engineering, 83 (25-26):21002111,2005

33. E.M. Yettou, A. Desrochers, and Y. Champoux. Experimental study on the water impact of a symmetrical wedge. Fluid Dynamics Research, 38(1):47-66, 2006.

34. W.H. Yuan, B. Wang, W. Zhang, Q. Jiang, and X.T. Feng. Development of an explicit smoothed particle finite element method for geotechnical applications. Computers and Geotechnics, 106:42-51, 2019.

35. W. Zhang, W.H. Yuan, and B. Dai. Smoothed particle finite-element method for largedeformation problems in geomechanics. International Journal of Geomechanics, 18(4):04018010, 2018
36. X. Zhang, E. Oñate, S.A.G. Torres, J. Bleyer, and K. Krabbenhoft. A unified lagrangian formulation for solid and fluid dynamics and its possibility for modelling submarine landslides and their consequences. Computer Methods in Applied Mechanics and Engineering, 343:314338, 2019.

37. Z.L. Zhang, T. Long, J.Z. Chang, and M.B. Liu. A smoothed particle element method (spem) for modeling fluid-structure interaction problems with large fluid deformations. Computer Methods In Applied Mechanics And Engineering, 356:261-293, 2019.

38. Z.Q. Zhang, G.R. Liu, and B.C. Khoo. Immersed smoothed finite element method for two dimensional fluid-structure interaction problems. International Journal of Numerical Methods in Engineering, 90:1292-1320, 2012.

39. W. Zheng and G.R. Liu. Smoothed finite element methods (s-fem): An overview and recent developments. Archives of Computational Methods in Engineering, 25:397-435, 2018. 